\title{
Appraisal of Instantaneous Phase-Based Functions in Adjoint Waveform Inversion
}

\author{
Clara Estela Jiménez Tejero ${ }^{\circledR}$, Valenti Sallares, and César R. Ranero
}

\begin{abstract}
Complex signal analysis allows separation of instantaneous envelope and phase of seismic waveforms. Seismic attributes have long routinely been used in geological interpretation and signal processing of seismic data as robust tools to highlight relevant characteristics of seismic waveforms. In the context of adjoint waveform inversion (AWI), it is crucial choosing an efficient parameter to describe the seismic data. The most straightforward option is using whole waveforms but the mixing of amplitude and phase parameters increases the nonlinearity inherent to the methodology. Several studies support the good functioning of the instantaneous phase (IP), a more linear parameter to measure the misfit between synthetic and recorded data. The IP is calculated using the inverse of the tangent function, where its principal value can be defined either wrapped in between different limits or also unwrapped. The wrapped phase presents phase jumps that reflect as noise in the inversion results. The conditioning of these discontinuities solves the problem partially and the continuous unwrapped IP is not a good descriptor of the waveform. For this reason, it is worth to explore beyond the traditional description of the IP parameter. Two alternative functions have been studied: 1) a revision of the triangular IP and 2) the first implementation of the normalized signal. The main objective of this paper is therefore, to review the fundamentals of the IP attribute in order to design robust IP-based objective functions which allow mitigating the inherent nonlinearity in the AWI method.
\end{abstract}

Index Terms-Acoustic propagation, backpropagation algorithms, cost function, finite difference methods, gradient methods, inverse problems, nonlinear equations, phase estimation.

\section{INTRODUCTION}

C OMPLEX signal analysis was introduced in the context of geological interpretation and geophysical exploration in the 1970s [23], [24]. Currently, it is a well-established seismic analytical method widely used both in academy and industry [1]. In complex signal analysis, the seismic attributes are called instantaneous because they produce local measures of the waveform at any given time, in contrast to the Fourier analysis that produces global measures in frequency domain. In complex signal analysis, the seismic trace is represented as the real part of the complex analytical signal,

Manuscript received July 28, 2017; revised October 25, 2017, December 18, 2017, and January 24, 2018; accepted February 26, 2018. This work was supported in part by Repsol under Project CODOS Fase-2, in part by the Spanish MINECO through Project FRAME under Grant CTM201571766-R, and in part by the Generalitat de Catalunya under Grant 2014 SGR 940. (Corresponding author: Clara Estela Jiménez Tejero.)

C. E. Jiménez Tejero and V. Sallarès are with the Barcelona Center for Subsurface Imaging, Institute of Marine Sciences, Spanish National Research Council, 08003 Barcelona, Spain (e-mail: ejimenez@icm.csic.es).

C. R. Ranero is with Institució Catalana de Recerca i Estudis Avançats, Spanish National Research Council, 08003 Barcelona, Spain.

Color versions of one or more of the figures in this paper are available online at $\mathrm{http}: / /$ ieeexplore.ieee.org.

Digital Object Identifier 10.1109/TGRS.2018.2811245
$f_{a}(t)=E(t) \cdot e^{j \theta}$ where the imaginary part is called direct quadrature (DQ) and is obtained as the signal phase shifted by $(\pi / 2)$ radians. The main instantaneous attributes are the instantaneous envelope (IE), $E(t)$, and the instantaneous phase (IP), $\theta(t)$, the two independent variables from which other attributes can be derived. To separate the IE and the IP information from the seismic waveform is, in fact, the main purpose of complex signal analysis. Instantaneous attributes describe well the waveform shape and are thus appropriate visual tools for seismic interpretation. On the one hand, the IE represents the instantaneous energy of the waveform, which is proportional to the reflection coefficient, and therefore capable of highlighting relevant seismic features, like bright spots, unconformities, major lithology boundaries, etc. Complementarily, IP helps to distinguish between the continuous and the discontinuous events, showing bedding accurately, representing all events equally as it has no amplitude information.

We apply the characteristics of the seismic complex trace analysis in the framework of adjoint waveform inversion (AWI). Proposed for over three decades by Lailly [16] and Tarantola [25], AWI is a data-fitting procedure that aims at retrieving detailed rock properties from the subsurface by comparing the recorded and synthetic seismograms. As opposed to travel time tomography techniques which only use the information from travel time differences from a limited set of seismic phases from each seismogram, AWI pursuits the minimization of objective functions (OFs) that compare waveform attributes between the recorded and the simulated seismograms, whose gradients are computed using the adjoint method. This makes the AWI a powerful tool to reconstruct models of physical properties of considerably higher resolution than travel time tomography methods, potentially of an increased resolution of up to one order of magnitude. It is suitable for all types of acquisition geometries and it is appropriate for marine and land data. It has so far been applied to a broad range of frequencies; long wavelengths (up to $0.1 \mathrm{~Hz}$ ) for regional studies with global earthquake records [3], [9], for active seismic source experiments for subsurface data at medium scale $(10 \mathrm{~Hz})$, and for the water column at small scale $(50 \mathrm{~Hz})$ [8] and even at ultrahigh frequency marine seismic reflection data $(1 \mathrm{kHz})$ [21]. Also, it allows multiparameter inversion; e.g., $p$ and $s$ wavevelocities, anisotropy, density, and attenuation. Nevertheless, AWI is not exempt from problems. In its more common version, where the $\mathrm{OF}$ to be minimized is the L2-norm between the recorded and the simulated seismograms, its success depends critically on having a seismogram that is not cycleskipped at the lowest inverted frequency, usually implying a 
TABLE I

Summary of Drawbacks in AWI: Problems (I), Causes (II), And Possible Solutions (III)

\begin{tabular}{|c|c|c|}
\hline I. Problem & II. Causes & III. Possible solutions \\
\hline $\begin{array}{l}\text { Undetermined system } \\
\text { (many local minima) }\end{array}$ & $\begin{array}{l}\text { - Highly non-linear method } \\
\text { - Cycle-skipping between } \\
\text { recorded and simulated data }\end{array}$ & $\begin{array}{l}\text { - Data processing (filtering, windowing ...) } \\
\text { - Multi-scaling technique } \\
\text { - Robust objective function/adjoint source } \\
\text { - Build accurate initial models using travel-time information: } \\
\text { - Using refraction-type, long-offset acquisition } \\
\text { geometry (e.g. long streamer, ocean bottom nodes) } \\
\text { - Redatuming of near-vertical reflection data } \\
\text { to the seafloor }\end{array}$ \\
\hline $\begin{array}{l}\text { High computational cost } \\
\text { and time consuming }\end{array}$ & $\begin{array}{l}\text { - Large amount of data } \\
\text { - Complex physical propagation } \\
\text { with numerous intervening parameters } \\
\text { - Optimization problem }\end{array}$ & $\begin{array}{l}\text { - High performance computing } \\
\text { - Multi-shooting } \\
\text { - Data pre-conditioning and decimation } \\
\text { - Identifying the most sensitive parameters } \\
\text { to the seismic attributes of interest } \\
\text { (e.g. acoustic vs elastic, isotropic vs anisotropic) } \\
\text { - In gradient-based method, gradient pre-conditioning } \\
\text { to reduce number of iterations } \\
\text { - Higher-order (e.g. Hessian-based) optimization techniques }\end{array}$ \\
\hline $\begin{array}{l}\text { Presence of noise } \\
\text { in real data }\end{array}$ & $\begin{array}{l}\text { - Noise screening important freq. } \\
\text { spectrum in the seismogram }\end{array}$ & - Data pre-conditioning and data redundancy \\
\hline
\end{tabular}

very good starting model [10]. An alternative strategy is to use a robust $\mathrm{OF}$ that is able to mitigate the strong nonlinearity of the method [3], [14], [17]. Table I gives a summary of the main issues of using AWI with the related causes and solutions. For a general overview of AWI and detailed description of its drawbacks, see [27].

A number of works show the instantaneous attributes as good candidates to perform as OFs in AWI [3], [6], [9], [14]. The IP does not contain amplitude information, and therefore, it presents a more linear behavior than the original signal, in which the amplitude and the phase are mixed. The IP is applied to AWI in [3] and [9] with earthquake records at large regional scale. The synthetic studies presented in [6] and [14] for active seismic data at local scale show that the instantaneous attributes are less sensitive to cycle skipping related issues than the L2-norm. Particularly, in [14], a modified version of the IP, which here we refer to as triangular IP, is shown to be more robust than the L2-norm, the IE, and the cross correlation travel time OFs. The principal value of the IP, which is obtained as the inverse of the tangent function, is typically calculated wrapped in AWI [3], [9]. When the principal value is wrapped, natural phase jumps emerge between the limits, producing spikes that, as we will show, pollute the inversion results. To solve this problem, one option is the filtering and/or the smoothing of the phase jumps, as it is done in the works mentioned earlier with global earthquake data [3], [9], where the IP is wrapped between $(-\pi, \pi)$. However, the smoothing of the IP consequently modifies the original parameter. Another way to avoid the undesirable phase jumps is to calculate the IP unwrapped, as it responds as a continuous function. There are no works in the literature where the unwrapped IP is applied in AWI as an OF. As we show in this paper, despite the absence of phase jumps due to its continuity, its linear character rather than periodic does not describe the waveform with sufficient detail.

In this paper, we explore the concept of the IP by analyzing the limitations and the benefits of the different domains to define its principal value. In particular, we analyze the IP wrapped between $(-\pi, \pi)$ and $(-(\pi / 2),(\pi / 2))$ and also unwrapped. In Section II, we present a general overview of the AWI applied in this paper and the technical details and parameters used in our synthetic tests. Next, we show how to extract the IE and the IP from the waveform using an empirical adaptive method called the Huang transform (Hu-T) [12] and how to assess the IP and the corresponding adjoint source (AS) in the context of AWI. As a forward step, we propose two more IP-based attributes with the main concern of producing continuous and periodic functions which do not need any further conditioning; the triangular IP, firstly presented in [14], and the phase carrier function (sine or cosine) that we refer as "normalized signal" OF, presented in this work for the first time. The ASs for the different IPbased OFs described in this paper differ from each other. The common feature among the different ASs functions is that they are composed in a similar way by two terms. However, although the first term can be calculated directly, the calculation of the second term increases the numerical complexity and the computational time. Thereby, a priority is to explore which of the OFs are robust in a realistic AWI scenario, favoring the strategies with a more simple numerical approach and that require less time calculation. The alternative OFs proposed, namely the triangular IP and the normalized signal, show high-resolution capacity and a robust behavior in the presence of cycle skipping, which mainly occurs when data lack low frequencies and/or a poor initial model is used. Also, they may be incorporated in hybrid strategies to 
provide appropriate initial models for the conventional L2norm approach.

\section{AdJOINT WAVEFORM INVERSION}

We implemented a time domain 2-D acoustic AWI with a gradient-based optimization scheme to extract the information from the active seismic synthetic data. We distinguish the main blocks.

1) Forward propagation: synthetic data, $u^{s}$, are generated by solving the wave equation in an initial model for the first iteration, and, for the following ones, the updated model obtained from the previous iteration is used. The homogeneous, isotropic, and acoustic approximations are applied to the wave equation, which is solved by finite differences

$$
\frac{1}{c^{2}} \ddot{u}(\mathbf{r}, t)-\nabla^{2} u(\mathbf{r}, t)=f(\mathbf{r}, t)
$$

where the velocity $c=((K / \rho))^{1 / 2}, K$ is the bulk modulus, and the value of the density is assumed to be constant $\rho=1000 \mathrm{~kg} / \mathrm{m}^{3}$. The factor $f$ is the acoustic source. The shot is obtained for each receiver position and time step, $u\left(\mathbf{r}\left(R_{i}\right), t_{j}\right)$. We have tested our inversion code using either $K$ or $c$ as inversion parameter. Using $K$, our code achieves a larger number of succesful iterations till higher frequency values compared to the case of using $c$ as inversion parameter. The better convergence accomplished when inverting $K$ parameter results in inversion images with better resolution. We interpret that the underlying reason is the smaller uncertainty in the calculation of $K$ gradient, $\mathrm{g}_{K}$, compared to the calculation of $c$ gradient, $g_{c} \approx c \cdot g_{K}$. Thus, we invert $K$ instead of $c$ as in other works in the literature, elastic [7], [15], or acoustic [25].

2) The misfit between the synthetic and the reference data is

$$
\chi=\sum_{i} \int_{t} M_{R_{i}}(t) d t
$$

where $M$ is the OF and the summation applies to all the receivers, $R_{i}$. In this paper, IP-based parameters are used to design the OFs, following the least-square criterion.

3) The model parameter $K$ must be updated toward the correct direction, where the misfit between the reference and the synthetic data decreases. The adjoint method [25] is used to compute the gradient of $K$ for each shot in the model, $g=\nabla_{K} \chi$, by convolution of the synthetic and adjoint wavefields, for each receiver

$$
g(\mathbf{r})=-\int_{t} \partial_{t} u_{s}(\mathbf{r}, t) \partial_{t} \psi(\mathbf{r}, T-t) d t
$$

where the adjoint field $\psi$ is obtained back propagating the AS in time for each receiver. The AS is calculated for each receiver as

$$
\mathbf{f}(t)=-\nabla_{u^{s}} M(t) .
$$

4) We use the nonlinear conjugate gradient method to find the step size toward the optimal direction for the decrease of the misfit. This methodology uses the gradient obtained in the previous iteration to obtain the search direction $\mathbf{d}_{i}[11]$

$$
\mathbf{d}_{i}=-g_{i}+\beta_{i} \mathbf{d}_{i-1} ; \quad \beta_{i}=\frac{g_{i}\left(g_{i}-g_{i-1}\right)}{\left|g_{i}\right|^{2}} .
$$

5) Finally, the parameter $K$ is updated as

$$
K_{i+1}=K_{i} \cdot e^{\alpha_{i} \cdot \mathbf{d}_{i}}
$$

and therefore, the $p$-wave velocity is $c_{i+1}=$ $\left(K_{i+1} / \rho\right)^{1 / 2}$. The optimal step $\alpha$ is calculated using a polynomial approximation around the minimum of the misfit [18]. This process is repeated till the synthetic data match the reference data. For more technical details on our code implementation, see [7] and [14].

\section{InSTANTANEOUS PHASE}

The IP of a time signal is computed in complex trace analysis as the phase of the complex analytical function, formed by the signal, $u(t)$, and its quadrature, $q(t)=\mathrm{DQ}[u(t)]$. The magnitude $\mathrm{DQ}[u(t)]$ is often calculated by applying the Hilbert Transform (Hi-T) [3]. The Hu-T, an adaptive data analysis proposed by Huang et al. [12], can also be applied. The advantage of using the Hu-T instead of solving the Hi-T lies not only in avoiding complicated numerical integration, but also it is proven to work better for non-linear and non-stationary data [12], [13]. We use the Hu-T to extract the IE from the signal. It is numerically efficient and can be easily applied to data of any kind; however, the only demand is that data must be monocomponent, i.e., data with a well-defined frequency. In this paper, we fulfill this requirement following a multiscale approach [5], applying a sufficiently narrow bandpass filter on the source and the data $(0.5 \mathrm{~Hz})$. Also, it is important to have an accurate interpolation method. The extraction of the IE from the signal is an iterative process that gives as a result the IE and also the normalized signal, $u_{n}$, in which all the minima and the maxima are -1 and +1 , respectively. The IE is built, at the first iteration, by interpolating all the maxima in the absolute value of the original signal. Then, the normalized signal is obtained in a second step as

$$
u_{n}^{i}(t)=\frac{u^{i}(t)}{E^{i}(t)} .
$$

However, this calculation rarely succeeds at the first attempt. Due to fluctuation errors of the interpolation, the extrema of the normalized signal might exceeds the limits of \pm 1 . For this reason, the correct values for $E(t)$ and $u_{n}(t)$ are generally obtained after a few iterations. The final IE uniquely defined for a particular receiver is obtained as

$$
E(t)=\frac{u(t)}{u_{n}(t)}, \quad\left|u_{n}(t)\right| \leq 1 .
$$

Once the IE is extracted, we must obtain the DQ of the normalized signal, as the normalized signal phase shifted $(\pi / 2)$ radians

$$
q_{n}(t)=D Q\left[u_{n}(t)\right]=u_{n}\left(\theta(t)-\frac{\pi}{2}\right) .
$$


TABLE II

DifFERENT CRITERIA To BUILD THE ANALYTICAL SignaL

\begin{tabular}{c|c|c|l}
\hline Cases & $\mathbf{u}_{n}(\mathbf{t})$ & $\mathbf{q}_{n}(\mathbf{t})$ & $f_{\text {an }}(t)=E(t) \cdot e^{j \theta(t)}$ \\
& & & \\
\hline $\mathrm{A}$ & $\sin \theta(t)$ & $-\cos \theta(t)$ & $E(t) \cdot\left(-q_{n}(t)+j \cdot u_{n}(t)\right)$ \\
$\mathrm{B}$ & $\cos \theta(t)$ & $\sin \theta(t)$ & $E(t) \cdot\left(u_{n}(t)+j \cdot q_{n}(t)\right)$ \\
\hline
\end{tabular}

The normalized seismic signal can be assumed to be a sine or a cosine type function, two criteria that we refer as case A and case $\mathrm{B}$, respectively. The corresponding normalized DQ and the complex analytical signal in each case is displayed in Table II.

Assuming that for our narrow bandpass of $0.5 \mathrm{~Hz}$, the frequency of the signal is a well-defined constant and positive value, $\theta(t) \approx \omega \cdot t$

$$
\frac{\partial \sin \theta(t)}{\partial t}=\omega \cdot \cos \theta(t) ; \quad \frac{\partial \cos \theta(t)}{\partial t}=-\omega \cdot \sin \theta(t)
$$

this leads to the following expression for the DQ (adding the IE) in both cases, A and B:

$$
q(t)=-\frac{E(t)}{\omega} \cdot \frac{\partial u_{n}(t)}{\partial t} .
$$

Even if $\omega$ value is unknown, we still can obtain the DQ normalizing the expression $-E(t) \cdot\left(\partial u_{n}(t) / \partial t\right)$ so that the maximum absolute value is 1 , as we are using the traces $u(t)$ and $q(t)$ normalized to 1 to calculate the misfit. ${ }^{1}$ However, to avoid numerical problems we apply the Pythagorean identity to obtain the absolute value of the quadrature as $E(t) \cdot\left(1-u_{n}(t)^{2}\right)^{1 / 2}$. We then use (11) to obtain the sign of the quadrature. The expression for $q(t)=\mathrm{DQ}[u(t)]$ is then rearranged as

$$
q(t)=\operatorname{sgn}\left(-\frac{\partial u_{n}(t)}{\partial t}\right) \cdot E(t) \cdot \sqrt{1-u_{n}(t)^{2}} .
$$

Finally, the IP is obtained as $\theta(t)=\operatorname{atan}((\sin \theta(t) / \cos \theta(t))$, for cases $\mathrm{A}$ and $\mathrm{B}$

$$
\begin{gathered}
\theta_{A}(t)=-\operatorname{atan}\left(\frac{u_{n}(t)}{q_{n}(t)}\right)=-\operatorname{atan}\left(\frac{u(t)}{q(t)}\right) \\
\theta_{B}(t)=\operatorname{atan}\left(\frac{q_{n}(t)}{u_{n}(t)}\right)=\operatorname{atan}\left(\frac{q(t)}{u(t)}\right) .
\end{gathered}
$$

Thus, the common characteristic between using the Hi-T and the Hu-T schemes is that the IP is finally extracted as the phase of the complex analytical signal $f_{\text {an }}(t)=E(t)$. $e^{j \theta(t)}$ [see Table II and (13) and (14)]. The difference lies in the procedure to estimate the DQ of the signal. In the Hi-T formalism, the DQ is computed directly by calculating the Hi-T of the original signal [3]. In our formalism, the

\footnotetext{
${ }^{1}$ Note that we are using the word "normalization" for two different types of normalizations. In the case of the original signal $u(t)$ and its quadrature $q(t)$, we use the classical normalization where just the maximum in the absolute value of the signal is 1 . In the case of the normalized signal $u_{n}(t)$ and normalized quadrature $q_{n}(t)$, it is a special type of normalization, calculated with the Hu-T iterative method, where each of the extrema reach the -1 or 1 value (depending if it is a minimum or a maximum).
}
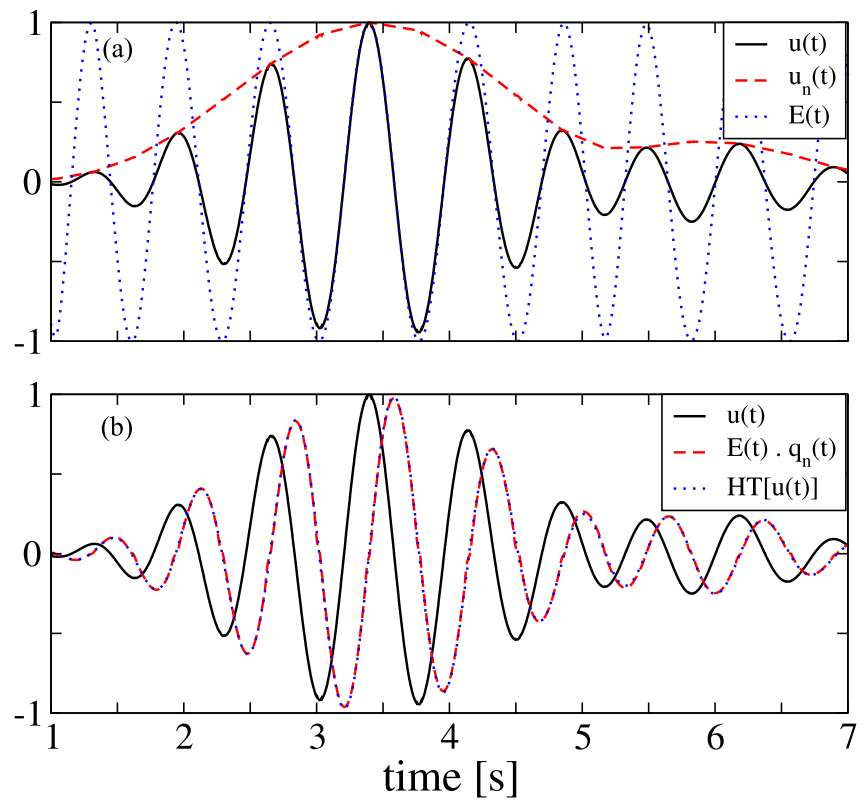

Fig. 1. Example of the extraction of the IE and normalized signal from monocomponent data. (a) Original waveform $u(t)$ (black solid line), the IE, $E(t)$ (red dashed line), and the normalized signal, $u_{n}(t)$ (blue dotted line) extracted with the Hu-T method. (b) Original waveform (solid black line) and the reconstructed quadrature $q(t)=\mathrm{DQ}[u(t)]$, using the Hu-T method $\mathrm{DQ}[u(t)]=\operatorname{HuT}[u(t)]$ (red dashed line) versus the DQ calculated with the Hi-T method DQ $[u(t)]=\operatorname{HiT}[u(t)]$ (blue dotted line).

Hu-T method is used first to extract numerically the IE from the signal. Then, the DQ is calculated using (12). To show the validity of our methodology, we display in Fig. 1(a) an example of a monocomponent signal and the final extracted IE and normalized signal. Fig. 1(b) shows the equivalent results obtained for the DQ of the signal reconstructed with the Hu-T method versus the DQ calculated using the Hi-T (using the Hi-T algorithm from the MATLAB library).

Despite that in the complex trace analysis only case B is usually considered, in this paper we also include case A. The IPs obtained in cases A (13) and B (14) are equivalent but shifted $\pi / 2$ radians with respect to each other. As we will show in the results section, we can give a good description of the waveform within each criterion, depending on the considered domain for the principal value. In Fig. 2, we plot, for cases A and $\mathrm{B}$, the principal value of the IP in the different domains where it can be defined: in Fig. 2(a), for the domain $(-\pi, \pi)$, in Fig. 2(b), for the domain $(-(\pi / 2),(\pi / 2))$, and in Fig. 2(c), for the unwrapped case. The IP is unwrapped in this paper using the IP wrapped between $(-\pi, \pi)$ and adding $2 \pi$ at each phase jump. Within a specific domain, the IP functions for case A are plotted in red dashed lines and in case B in red dotted lines. The IPs wrapped between $(-\pi, \pi)$ have the same periodicity as the signal and half of the periodicity in case of the IPs wrapped between $(-(\pi / 2),(\pi / 2))$. The OF of the IP at each receiver is defined as

$$
M_{r}(t)=\frac{\Delta \theta^{2}(t)}{2}=\frac{\left(\theta^{s}(t)-\theta^{o}(t)\right)^{2}}{2} .
$$

The corresponding AS must be calculated as

$$
\mathbf{f}_{\mathbf{r}}(t)=-\Delta \theta \cdot \frac{\partial \theta^{s}(t)}{\partial u^{s}(t)}
$$


(a)

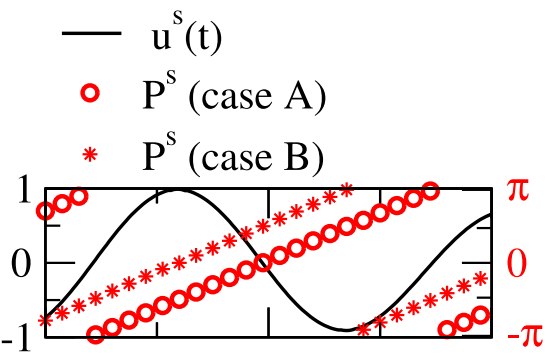

(b)

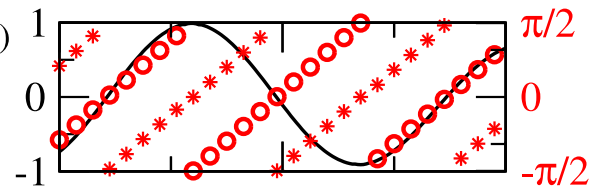

(c)

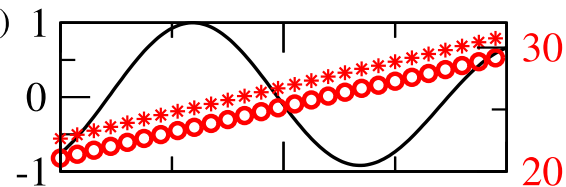

(d)

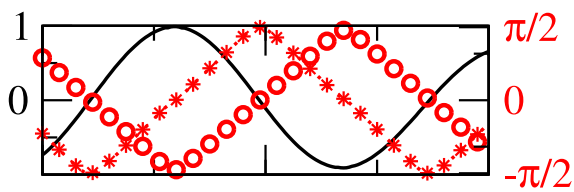

(e)

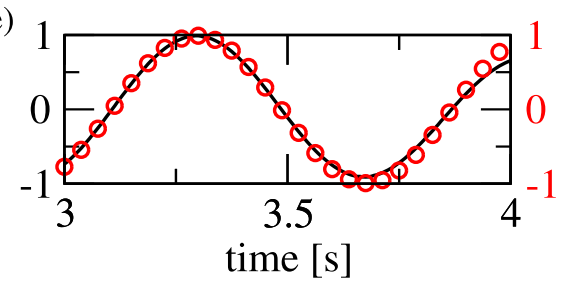

Fig. 2. Example of a waveform (black line) and the corresponding IP-based parameters represented with points (red circles for case A and red stars for case B). The figure is shown for just $1 \mathrm{~s}$ in the $x$-axis to highlight the differences. (a) IP wrapped between $(-\pi, \pi)$. (b) IP wrapped between $(-(\pi / 2),(\pi / 2))$. (c) Unwrapped IP. (d) Triangular IP. (e) Normalized signal.

where $\partial \theta$ is equal for cases $\mathrm{A}$ and $\mathrm{B}$

$$
\partial \theta_{A}=\partial \theta_{B}=\frac{-q(t) \cdot \partial u(t)+u(t) \cdot \partial q(t)}{E(t)^{2}} .
$$

Taking into account the coincident results of the quadrature $q(t)=\mathrm{DQ}[u(t)]$ for monocomponent data using the Hu-T and Hi-T methods in Fig. 1(a), panel II, we can apply the expression defined for the Hi-T in [3] to extract the derivative $\partial u$ from the second term $u(t) \cdot \partial q(t)$ in the expression above, as

$$
f(t) \cdot \partial \mathrm{DQ}[u(t)]=-\partial u(t) \cdot \mathrm{DQ}[f(t)]
$$

this leads to the final expression of the AS at each receiver

$$
\mathbf{f}_{\mathbf{r}}(t)_{A, B}=\frac{\Delta \theta_{A, B}(t) \cdot q^{s}(t)}{E^{s}(t)^{2}}+\mathrm{DQ}\left[\frac{\Delta \theta_{A, B}(t) \cdot u^{s}(t)}{E^{s}(t)^{2}}\right] .
$$

In Fig. 7 , both terms in the $\mathrm{AS}, T_{1}$ and $T_{2}$, are plotted in the first and the second columns, respectively, for cases A (black solid line) and B (red dashed line), respectively. The $T_{1}$ and $T_{2}$ terms show clear differences for the domains $(-\pi, \pi)$ [Fig. 7(a)] and $(-(\pi / 2),(\pi / 2))$ [Fig. 7(b)], and also for cases A and B. In the case of the unwrapped IP [Fig. 2(c)] and due to its linear character, the phase difference for cases $\mathrm{A}$ and $\mathrm{B}, \Delta \theta_{A}$ and $\Delta \theta_{B}$, is equivalent and therefore, the terms $T_{1}$ and $T_{2}$ as well [see Fig. 7(c)]. The unwrapping of the IP is a solution to obtain a continuous function without phase jumps and without the need of a smoothing filter. As it is shown in Fig. 7(c), the unwrapped IP produces periodic and smooth AS terms. However, as we will show in the results of section VII, the unwrapped IP is not an optimal OF for AWI.

\section{Triangular Instantaneous Phase}

The IP holds the capability to operate well in AWI. However, the natural phase jumps between the limits of the wrapped principal values producing numerical instabilities and this produces noisy inversion results, as we show in Section VI (Fig. 6). The smoothing of the AS solves this problem; however, it produces certain loss in the resolution capacity. It is also possible to design a specific instantaneous wrapped IP function with no phase jumps that does not need any conditioning. The technique consists of multiplying the IP expression by the sign of the term contained in the denominator of the arctangent (the cosine). By doing this, we obtain a pseudophase signal that follows a continuous triangular shape. We use the definition domain of the IP wrapped between $(-(\pi / 2),(\pi / 2))$ because after the proposed modification, it has the same periodicity as the original signal, so it mimics better the waveform. We refer to this modified version of the IP as triangular IP signal, whose expressions, in cases $\mathrm{A}$ and $\mathrm{B}$ are

$$
\tilde{\theta}_{A}=\theta_{A} \cdot \operatorname{sgn}\left[q_{n}\right] ; \quad \tilde{\theta}_{B}=\theta_{B} \cdot \operatorname{sgn}\left[u_{n}\right] .
$$

Operating as in the former section, the corresponding misfit at each receiver $r$ is

$$
\tilde{M}_{r}(t)=\frac{\Delta \tilde{\theta}_{A, B}^{2}(t)}{2} .
$$

To obtain the AS, first we calculate the partial derivatives of the triangular IP as

$$
\begin{aligned}
& \partial \tilde{\theta}_{A}=\operatorname{sgn}\left[q_{n}\right] \cdot \partial \theta_{A}+\theta_{A} \cdot \partial \operatorname{sgn}\left[q_{n}\right] \\
& \partial \tilde{\theta}_{A}=\operatorname{sgn}\left[u_{n}\right] \cdot \partial \theta_{B}+\theta_{B} \cdot \partial \operatorname{sgn}\left[u_{n}\right] .
\end{aligned}
$$

The second term is neglected in both expressions as its value is 0 except for $q_{n}(t)=0$ (case A) and $u_{n}(t)=0$ (case B), where it is not defined. Thus, reorganizing the formula, the AS for the triangular IP at each receiver corresponds to

$$
\begin{aligned}
& \tilde{\mathbf{f}}_{r}(t)_{A} \\
& \quad=\frac{\Delta \tilde{\theta}_{A}(t) \cdot\left|q^{s}(t)\right|}{E^{s}(t)^{2}}+D Q\left[\frac{\Delta \tilde{\theta}_{A}(t) \cdot u^{s}(t) \cdot \operatorname{sgn}\left[q^{s}(t)\right]}{E^{s}(t)^{2}}\right]
\end{aligned}
$$

$$
\begin{aligned}
& \tilde{\mathbf{f}}_{r}(t)_{B} \\
& \quad=\frac{\Delta \tilde{\theta}_{B}(t) \cdot q^{s}(t) \cdot \operatorname{sgn}\left[u^{s}(t)\right]}{E^{s}(t)^{2}}+D Q\left[\frac{\Delta \tilde{\theta}_{B}(t) \cdot\left|u^{s}(t)\right|}{E^{s}(t)^{2}}\right] .
\end{aligned}
$$


Fig. 2(d) shows an example of a signal and the corresponding triangular IP for cases A and B. The triangular IPs and the original signal have the same periodicity in time. It should be noted that the time location of the extrema in the triangular IP in case A are coincident with the extrema of the original signal, although the signals appear shifted $\pi$ rad with respect to each other. Fig. 7(d) shows the first and second terms of the AS of the triangular IP. The triangular IP for case A was firstly presented in [14] and also recently applied in [21].

\section{Normalized Signal}

Another way to obtain a continuous and periodic measure of the IP is using the normalized signal $u_{n}(t)$ as parameter, i.e., the phase carrier function. As it was defined earlier, the normalized signal is the original signal modified so that each maximum and minimum are located to +1 and -1 , respectively. It is an indirect measure of the IP which plays the role of the sine/cosine of the IP. The cosine of the IP is also used in geological interpretation as a seismic processing tool [22], [28]. As in the case of the triangular IP function presented earlier, the cosine of the IP has no amplitude information and it does not introduce numerical spikes. Thus, the $\mathrm{OF}$ at each receiver is referred here as normalized signal

$$
M_{r}(t)=\frac{\Delta u_{n}^{2}(t)}{2}
$$

and as in the former cases, to obtain the AS

$$
\partial M_{r}(t)=\Delta u_{n}(t) \cdot \partial u_{n}(t) .
$$

The partial derivative $\partial u_{n}(t)$ can be obtained considering the term $u_{n}(t)$ as the carrier phase function, which either in the sine-type (case A) or cosine-type (case B) criteria corresponds to the same expression

$$
\partial u_{n}(t)=\frac{\partial u_{n}(t)}{\partial \theta} \partial \theta=-q_{n} \partial \theta .
$$

Also, the term can be expressed as $u_{n}(t)=(u(t) / E(t))$, where $E(t)=\left(u(t)^{2}+q(t)^{2}\right)^{1 / 2}$

$$
\partial u_{n}(t)=\frac{\partial u_{n}(t)}{\partial u(t)} \partial u(t)+\frac{\partial u_{n}(t)}{\partial E(t)} \partial E(t) .
$$

In both cases, the result for the partial derivative term is equivalent

$$
\partial u_{n}(t)=\frac{q_{n}(t)^{2}}{E(t)} \cdot \partial u(t)-\frac{u_{n}(t) \cdot q_{n}(t)}{E(t)} \cdot \partial q(t) .
$$

Operating as in the former cases, the AS for the normalized signal at each receiver corresponds to

$$
\tilde{\mathbf{f}}_{r}(t)=-\frac{\Delta u_{n}(t) \cdot q_{n}^{s}(t)^{2}}{E^{s}(t)}-\mathrm{DQ}\left[\frac{\Delta u_{n}(t) \cdot u_{n}^{s}(t) \cdot q_{n}^{s}(t)}{E^{s}(t)}\right] .
$$

The normalized signal parameter is shown in Fig. 2(e) and is compared to the original signal. Also, Fig. 7(e) shows the first and second terms of its AS. The similar shape of the $T_{1}$ term of the AS between the triangular IP in case A [black line in the first column, Fig 7(d)] and the normalized function [first column, Fig. 7(e)] is noticeable; therefore, similar inversion results are expected. In contrast to the IP parameters defined earlier, the original IP and the triangular IP, the boundary limits of the normalized signal parameter are naturally and uniquely defined. Besides, there is no distinction between cases $\mathrm{A}$ and $\mathrm{B}$.

\section{NUMERICAL CONSIDERATIONS}

1) The calculation of the second term $T_{2}$ of the AS function requires to obtain the DQ of the following expressions.

a) For the IP: $\left(\Delta \theta_{A, B}(t) \cdot u^{s}(t) / E^{s}(t)^{2}\right)$.

b) For the Triangular Phase:
i) Case A: $\left(\Delta \tilde{\theta}_{A}(t) \cdot u^{s}(t) \cdot \operatorname{sgn}\left[q^{s}(t)\right] / E^{s}(t)^{2}\right)$.
ii) Case B: $\left(\Delta \tilde{\theta}_{B}(t) \cdot\left|u^{s}(t)\right| / E^{s}(t)^{2}\right)$.
c) For the Normalized Signal:
$\left(\Delta u_{n}(t) \cdot u_{n}^{s}(t) \cdot q_{n}^{s}(t) / E^{s}(t)\right)$.

This cannot be done using the Hu-T directly, as the expressions are commonly a nonmonocomponent signal; however, they can be transformed. The nonmonocomponent data is assumed to be composed of different coexisting oscillatory waves with different frequencies. These oscillations called intrinsic mode functions (IMFs) are monocomponent signals. The extraction of the different IMFs of any data set is referred as the empirical mode decomposition (EMD) method, and it is explained in detail in [12] and [13]. As a summary of this process, the lower (upper) IE is calculated by connecting the minima (maxima) in the signal, respectively. Afterward, the average IE is obtained and this quantity is subtracted from the data. The process is repeated till this subtracted value achieves the definition of an IMF. To obtain the second IMF, this residual is treated as the new signal, and the same procedure outlined earlier is repeated for the new signal until all the IMFs are obtained. At the end of the process, the data is decomposed into its different IMFs as

$$
u=\sum_{i} \mathrm{IMF}_{i}+r
$$

where the remnant $r$ is normally a constant or a function with a single maximum or minimum. Once the IMFs are extracted, the Hu-T can be applied to each of them to obtain the instantaneous parameters IE, IP, normalized signal, and DQ. In our case, our data are generated with a narrow bandpass width of $0.5 \mathrm{~Hz}$. This allows to simplify numerically the extraction of the modes, as $T_{2}$ can be reproduced by just two IMFs, in which $r$ is reabsorbed inside the IMFs. Fig. 3 shows an example of the extraction of the IMFs from a nonmonocomponent signal. Fig. 3(a) shows the signal and the sum of the two IMFs extracted, which give the value of the original signal. In Fig. 3(b), the IMFs are plotted separately and in Fig. 3(c), we show the DQ of the signal, calculated as the summation of the DQs of each IMF, versus the DQ calculated using directly the Hi-T over the nonmonocomponent signal. In this respect, the quadrature obtained with the $\mathrm{Hu}-\mathrm{T}$ and the 

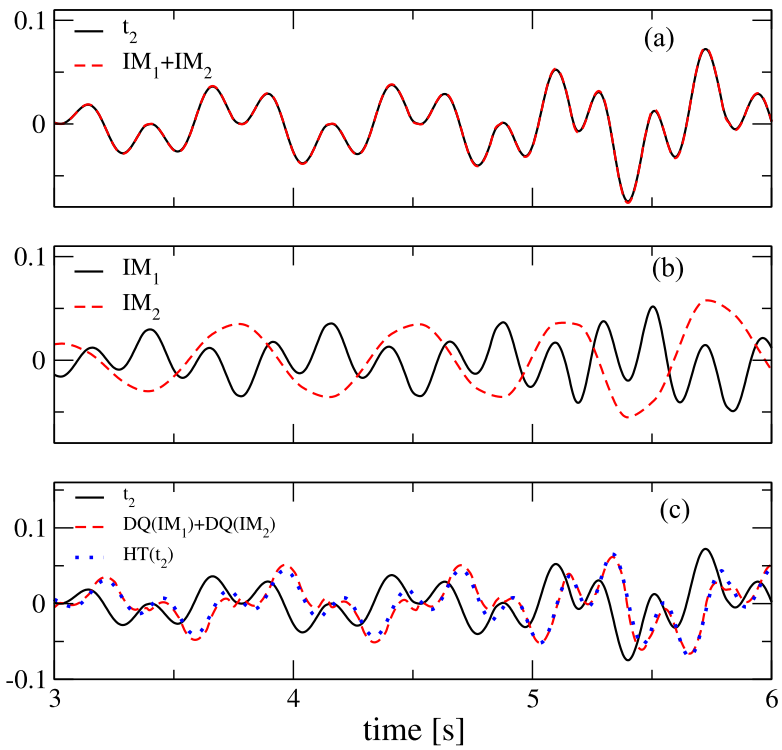

Fig. 3. Example of the extraction of the IMFs from nonmonocomponent data. (a) Monocomponent data (black line) and the summation of the extracted intrinsic modes $\mathrm{IM}_{1}+\mathrm{IM}_{2}$ (red dashed line). (b) Intrinsic modes $\mathrm{IM}_{1}$ (black solid line) and $\mathrm{IM}_{2}$ (red dashed line). (c) Nonmonocomponent data $t_{2}$ (black solid line) and its DQ calculated as a summation of the individual DQs for each intrinsic mode using the Hu-T method (red dashed line) versus the DQ calculated directly over the nonmonocomponent data using the Hi-T method (blue dotted line). The $t_{2}$ nonmonocomponent data refers to the term $T_{2}$ in the ASs, before calculating its DQ.

Hi-T is remarkably similar, differing only in the amplitude of certain peaks. Note that when both methods are applied to obtain the quadrature of a monocomponent signal, shown in Fig. 1(b), results are equivalent, and as was mentioned earlier, this justifies the use of (18) to obtain the final AS function. As a summary, in Fig. 4, we show the workflow to obtain the OF and the AS of a monocomponent signal using numerical adaptive data methods, like the Hu-T and EMD applied here.

2) As was already outlined in Section I, the wrapped IP presents phase jumps that produce numerical discontinuities also in the AS. These spikes reflect in the gradient and this distorts the search direction to update the velocity model. As we show in Fig. 6(b) [(iii) and (iv)], this produces noise in the inversion result. To avoid these numerical spikes, we apply a smoothing filter to the wrapped IP. In Fig. 6(a), we show an example of this smoothing applied to the IP wrapped between $(-(\pi / 2),(\pi / 2))$ and in Fig. 6(c) [(iii) and (iv)], the result of the inversion in which the numerical discontinuities have vanished.

3) To avoid discontinuities in the AS when $E^{s}(t)=0$, a constant quantity $\delta$ can be added to the IE in the denominator as $E^{s}(t): E^{s}(t)+\delta$. The higher this value is, the softer is the AS function at certain peaks. However, this does not alter the inversion results. Taking into account that $\max [E(t)]=1$, we have tried $\delta$ values from the range $\delta=10^{-5}$ to 1 . Using any $\delta$ value in the mentioned range and after 30 iterations during the first frequency step, we checked that the decrease of the misfit and the rms value differ at the most

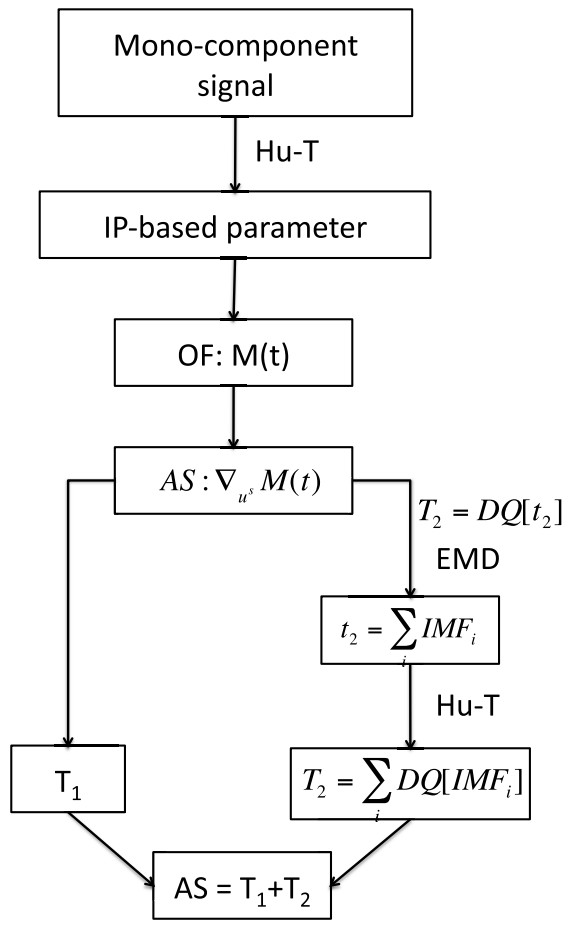

Fig. 4. Workflow for the calculation of the OF and AS for the IP-based parameters presented.

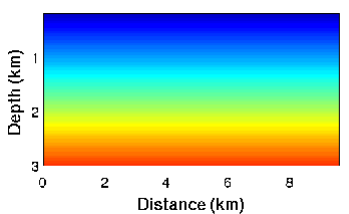

(a)

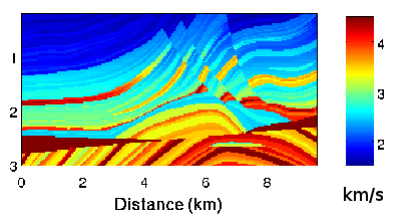

(b)
Fig. 5. (a) Initial model. (b) Reference "true" model.

by $3 \%$. Consequently, we fixed this parameter arbitrarily to $\delta=0.1$.

\section{INVERSION TESTS USING INSTANTANEOUS Phase-BAsed ObJective Functions}

\section{A. Test Parameters}

Similar to [14], we use the Marmousi model as benchmark [see Fig. 5(b)]. Therefore, to obtain the reference data from the Marmousi model, $u^{o}(t)$, forward propagation is also used. A Ricker wavelet with central frequency $f_{0}=20 \mathrm{~Hz}$ is used as a source. For the first iteration, we use a homogeneous vertical gradient velocity model displayed in Fig. 5(a). The space is discretized with a staggered grid [26], with $25 \mathrm{~m}$ spacing both in $x$ and $z$ directions and the simulation time is $8 \mathrm{~s}$. Along the $x$-direction, we locate a receiver at each point of the model and 16 sources equally spaced along the surface. We apply convolutional perfectly matched layers scheme [20] on the left, bottom, and right boundaries to avoid numerical reflections and a free surface at the top of the Marmousi model. As explained earlier, the width of the bandpass in the multiscale approach is kept fixed to $0.5 \mathrm{~Hz}$ so that the data preserves its monocomponent character. 


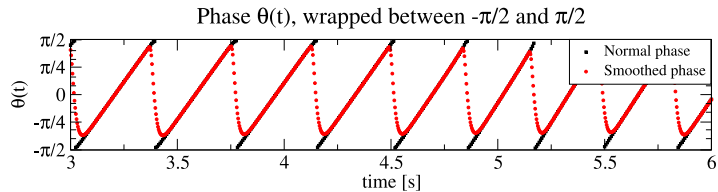

(a)
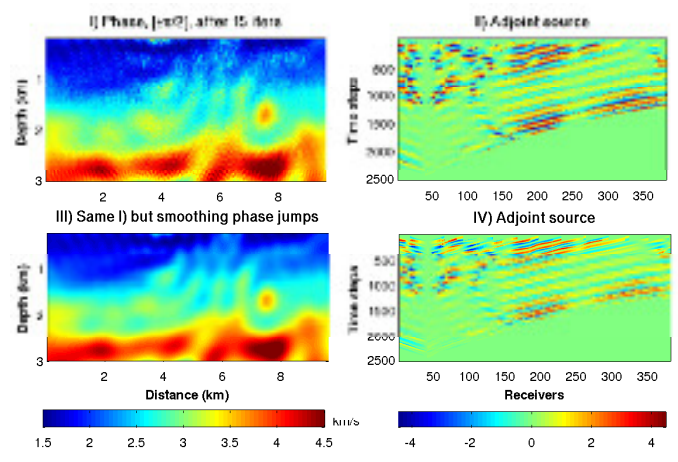

(b)

Fig. 6. (a) Plot of the IP wrapped between $\left(-\frac{\pi}{2}, \frac{\pi}{2}\right)$ (black squares) and the same when a smoothing filter is applied (red circles). (b) Inversion result (I) and AS (II) using the IP wrapped between $\left(-\frac{\pi}{2}, \frac{\pi}{2}\right)$ and the same in (III) and (IV) but using the smoothed IP. In both cases, the AS is shown for all the receivers and time steps, just for the first shot in the first iteration.

This makes the calculation of the instantaneous parameters using the Hu-T method straightforward. The total number of iterations per frequency step is fixed to 20 and the maximum frequency inverted is $12 \mathrm{~Hz}$. The lower frequency is $1 \mathrm{~Hz}$ to simulate data with low-frequency content and $4 \mathrm{~Hz}$ to test the algorithms for data with lack of low frequencies. The limits of the bandpass filter in the multiscale approach increase $0.5 \mathrm{~Hz}$ at every frequency step as follows: $\left(f_{0}, f_{0}+0.5\right),\left(f_{0}+\right.$ $\left.0.5, f_{0}+1\right),\left(f_{0}+1, f_{0}+1.5\right), \ldots$ etc. We evaluate the misfit between the reference and the simulated data at each iteration. The OFs evaluated and compared are the IP calculated with the inverse tangent function in its different domains, as well as the triangular IP, and the normalized signal.

\section{B. Inversion Tests}

In Fig. 8, we plot the inversion results for data containing low frequencies (starting frequency is $1 \mathrm{~Hz}$ ) and for the different IP parameters defined in an specific angular domain and criteria:

1) The direct calculation of the IP through the inverse of the tangent is as follows.

a) IP wrapped between $(-\pi, \pi)$, case A [Fig. 8(a)] and case B [Fig. 8(b)];

b) IP wrapped between $(-(\pi / 2),(\pi / 2))$, case $\mathrm{A}$ [Fig. 8(c)] and case B [Fig. 8(d)];

c) IP unwrapped [Fig. 8(e)].

2) The triangular IP for case A [Fig. 8(f)] and B [Fig. 8(g)].

3) The normalized signal [Fig. 8(h)].

Each row displays three types of inversion results plotted in three different columns. We want to explore the dependence of results by using separately the terms that confirm the AS

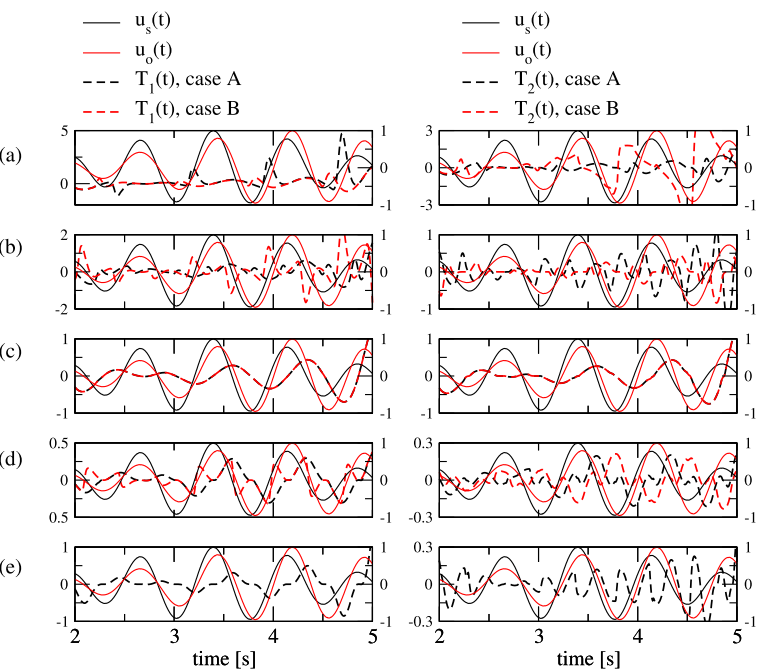

Fig. 7. First term $T_{1}(t)$ (left column) and second term $T_{2}(t)$ (right column) of the AS of the IP-parameters for the A (black dashed line) and B (red dashed line) criteria. The normalized synthetic and observed waveforms are also plotted as a reference (black and red solid lines). The $y$-axis for $T_{1}$ and $T_{2}$ is located on the left-hand side and for the waveforms is located on the righthand side of each panel. (a) and (b) Wrapped IPs are defined between $(-\pi, \pi)$ and $\left(-\frac{\pi}{2}, \frac{\pi}{2}\right)$, respectively, and smoothed to avoid numerical problems. (c) IP is unwrapped. (d) AS terms for the triangular IP. (e) Normalized signal.

and also using the complete expression. If $T_{1}$ and $T_{2}$ provide complementary information that improves the results, then both terms are necessary. By the contrary, if they provide analogous information or one of them can be shown to worsen the results, using only one term would simplify the calculations. The second term $T_{2}$ is more complex numerically. It increases the computational time as it implies the calculation of the IMFs of a nonmonocomponent signal, as explained earlier (see Fig. 4). Thus, we will favor the OFs which give good results using the $T_{1}$ term alone. The first column shows the result obtained when only the first term of the corresponding AS is used, $T_{1}$. The second column shows the results when the second term, $T_{2}$, is used, and the inversion result with both terms in the AS, $T_{1}+T_{2}$, is plotted in the third column. The inversion results of the wrapped IP are plotted in Fig. 8(a)-(d), and are obtained using the smoothed wrapped IPs in every case, in order to avoid the phase jumps which cause numerical spikes. For the IP wrapped between $(-\pi, \pi)$, the inversions generated with the $T_{2}$ term are better than with the $T_{1}$, in both cases A [Fig. 8(a)] and B [Fig. 8(b)]. Using the complete AS, the results worsen dramatically due to the influence of the $T_{1}$ term in case A [Fig. 8(a)]. When the IP is wrapped between $(-(\pi / 2),(\pi / 2))$, the inversion is only successful in case A [Fig. 8(c)].

Despite of the continuous and periodic behavior of the AS terms of the unwrapped IP, the inversion results are strikingly poor [Fig. 8(e)]. The three inversions are almost identical, consistent with the AS, which is equivalent for the terms $T_{1}$ and $T_{2}$. For the rest of the inversions in the different IP cases and criteria, there is a variety of results. The best inversion results are obtained using the triangular IP (case A) and the normalized signal; in particular, using the term $T_{1}$ in both cases [Fig. 8, first column of $(\mathrm{g})$ and $(\mathrm{h})]$. In these cases, the term 


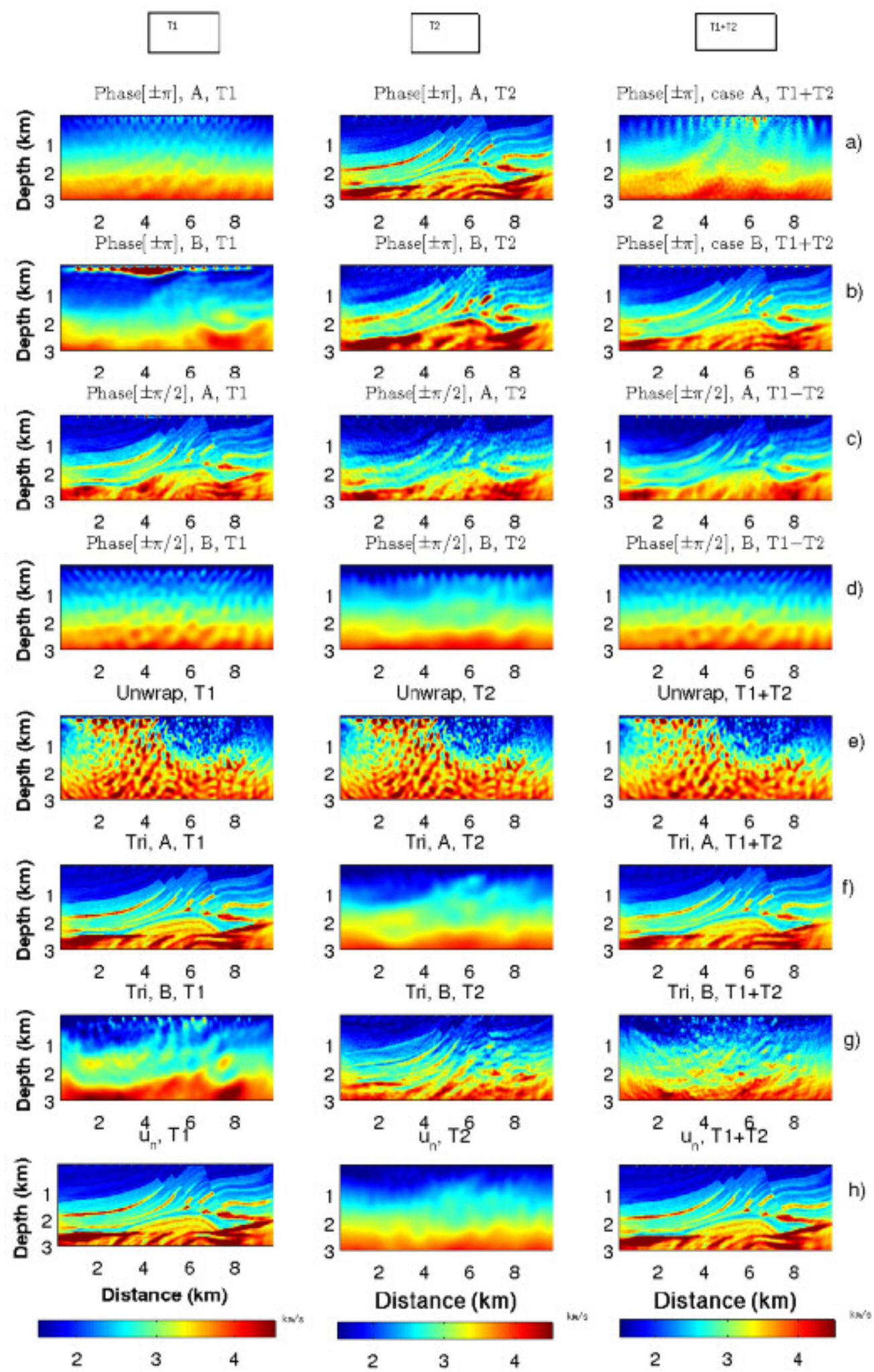

Fig. 8. Inversion results using $T_{1}$ (first column) and $T_{2}$ (second column) terms of the AS separately and using the complete expression $\mathrm{T}_{1}+T_{2}$ (third column), for the different IP criteria. (a) Inversion results when using the IP wrapped between $(-\pi, \pi)$ for case A. (b) Inversion results when using the IP wrapped between $(-\pi, \pi)$ for case B. (c) Inversion results when using the IP wrapped between $(-(\pi / 2),(\pi / 2))$ for case A. (d) Inversion results when using the IP wrapped between $(-(\pi / 2),(\pi / 2))$ for case B. (e) Inversion results when using the unwrapped IP. (f) Inversion results when using the triangular IP for case A. (g) Inversion results when using the triangular IP for case B. (h) Inversion results when using the triangular IP for the normalized signal.

$T_{2}$ is small compared with $T_{1}$ so it barely influences the result [see Fig. 7(d) and (e)]. The time cost to calculate the term $T_{1}$ for all the receivers and time steps is $1 \mathrm{~s}$, mainly due to calculation of the synthetic and reference IP. The calculation of both terms $T_{1}+T_{2}$ takes $2 \mathrm{~s}$, due to the calculation of the intrinsic modes. With respect to the time cost per iteration 


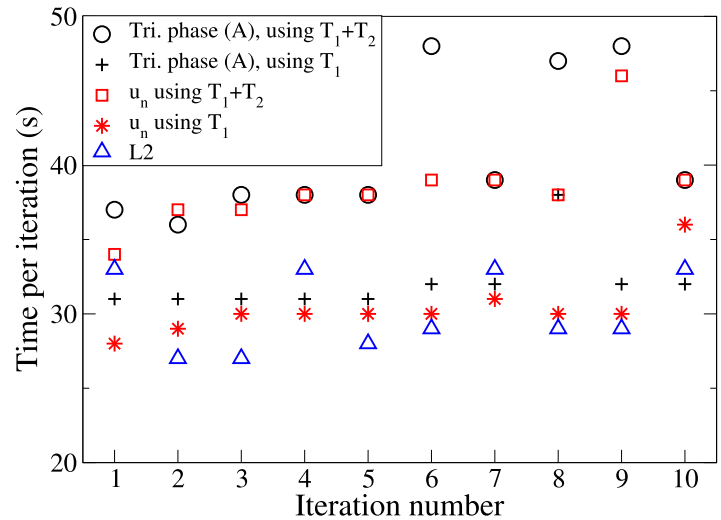

Fig. 9. Time cost per iteration for the first 10 iterations for the best strategies shown in Fig. 8: triangular phase (case A) for the complete AS (black circles) and using only $T_{1}$ (black plus) and normalized signal for the complete AS (red squares) and using only $T_{1}$ (red stars). The time cost for the L2 function is also shown as reference (blue triangles).

for an IP-based OF using the complete AS can increase from $25 \%$ to $60 \%$ compared with using the $T_{1}$ term alone. The time cost per iteration during the first 10 iterations is shown in Fig. 9 for the mentioned best strategies from Fig. 8. As a reference, the calculation with the L2 function is also included in the figure [the L2 inversion results are shown in Fig. 10(k) and (1)]. Also, it can be seen that the time cost per iteration for the IP-based functions using only the $T_{1}$ term in the AS is similar to the time cost for the straightforward L2 function. On top of that, the $T_{2}$ term is highly nonlinear, and whether we use the $\mathrm{Hu}-\mathrm{T}$ or the Hi-T, a wrong calculation would increase the uncertainty in the results, especially for noisy data. Taking into account this scenario, whenever the $T_{2}$ term does not improve the results such as in the mentioned cases, we will avoid its calculations using the term $T_{1}$ alone, therefore saving time and numerical problems. As a summary, the best results from Fig. 8 are plotted in the first column in Fig. 10, together with the velocity difference of each inversion compared with the reference model, which is shown in the second column. We have included as well in the last row the results for the waveform difference, the L2 OF. For each row, it has the following.

1) Fig. $10(a)$ and $(b)$ : IP wrapped $(-\pi, \pi)$, case A, with $T_{2}$.

2) Fig. 10(c) and $(d)$ : IP wrapped $(-\pi, \pi)$, case B, with $T_{1}+T_{2}$.

3) Fig. 10(e) and $(f)$ : IP wrapped $(-(\pi / 2),(\pi / 2))$, case A, with $T_{1}$.

4) Fig. $10(g)$ and $(h)$ : triangular IP, case A, with $T_{1}$.

5) Fig. 10(i) and $(j)$ : normalized signal, with $T_{1}$.

6) Fig. 10(k) and (l): L2.

In all cases, the reference model is well recovered in the top half of the model; however, the best inversions correspond to the triangular IP and the normalized signal OFs.

\section{Lack of Low Frequencies}

The seismic records typically acquired in most explorationtype surveys lack energy at low frequencies. In general, signalto-noise ratio is too small to retrieve usable signal below (a)

(c)

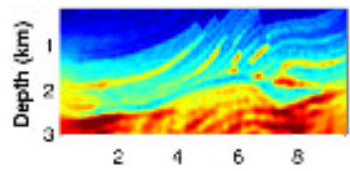

(e)

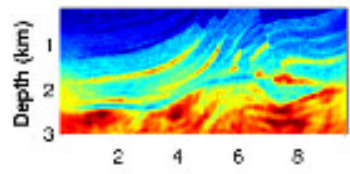

(g)

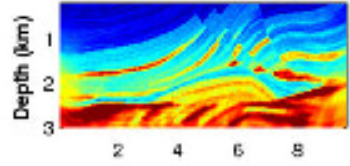

(i)

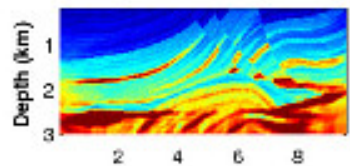

$(\mathrm{k})$

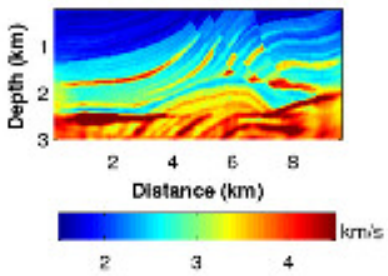

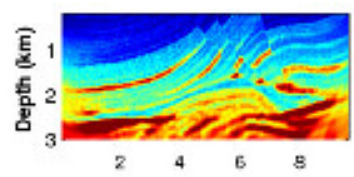

(b)

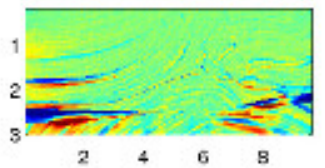

(d)

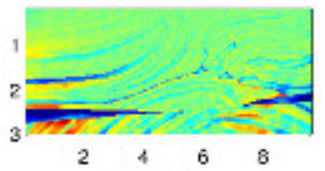

(f)

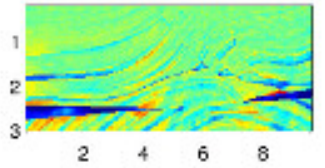

(h)

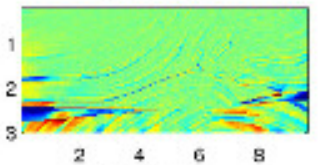

(j)

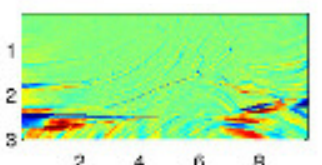

(1)

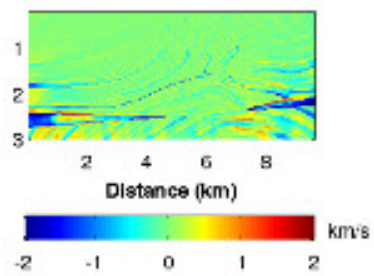

Fig. 10. Results selected from Fig. 8 (first column) and velocity difference with respect to the reference model (second column). (a) and (b) Inversion results for the IP wrapped between $(-\pi, \pi)$ using only $T_{2}$ of the AS for case A. (c) and (d) Inversion results for the IP wrapped between $(-\pi, \pi)$ using $T_{+} T_{2}$, case B. (e) and (f) Inversion results for the IP wrapped between $(-(\pi / 2),(\pi / 2))$ using only $T_{1}$, case A. (g) and (h) Inversion results for the triangular IP using only $T_{1}$, case A. (i) and (j) Inversion results for the normalized signal using only $T_{1}$. (k) and (1) Inversion results for the L2 OF.

3-4 Hz. To simulate the realistic conditions of data lacking low frequency signal, we filtered out in our source and data frequencies below $4 \mathrm{~Hz}$. The same cases shown in Fig. 10 are plotted in Fig. 11 for data lacking low frequencies below $4 \mathrm{~Hz}$. In this case, the multiscale approach from lower to higher frequency is [4-4.5], [4.5-5] Hz, ... etc. Only the inversion results generated with the triangular IP and the normalized signal OFs are robust enough to overcome nonlinearity and obtain acceptable results. Compared to Fig. 10, the velocity difference shows a larger error in location of the horizontal layers of the model. Also, the deeper part of the inverted model is resolved worst. As stated in the introduction, the most popular OF used in AWI with exploration-type data [27] is the L2-norm. It is simple, fast to calculate, and free of numerical problems. However, using the original signals to build the OF, the nonlinearity inherent in the AWI method increases due to the mixing of amplitude and phase. As a consequence, 
(a)

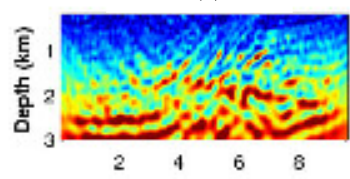

(c)

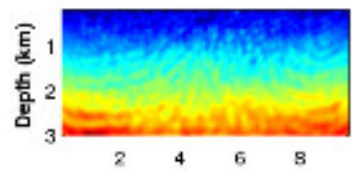

(e)

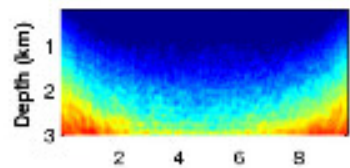

(g)

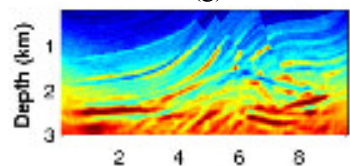

(i)

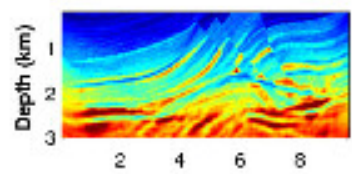

$(\mathrm{k})$

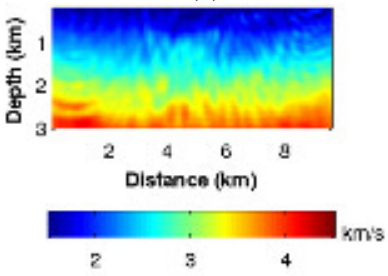

(b)

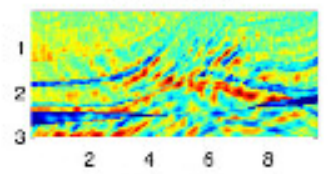

(d)

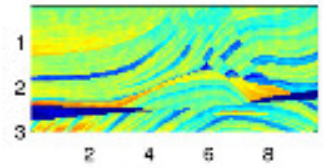

(f)

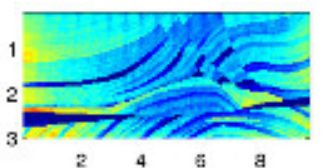

(h)

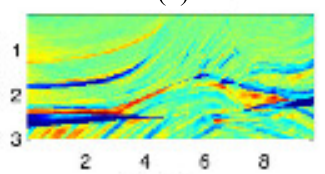

(j)

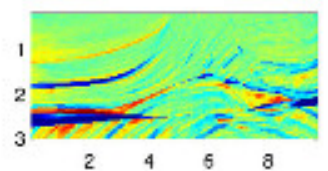

(1)

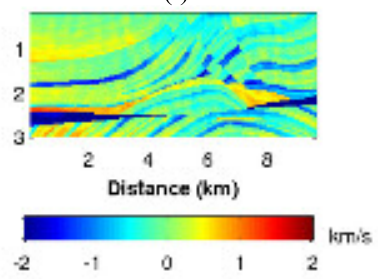

Fig. 11. Inversion results for the same strategies shown in Fig. 10 but without low frequencies. (a)

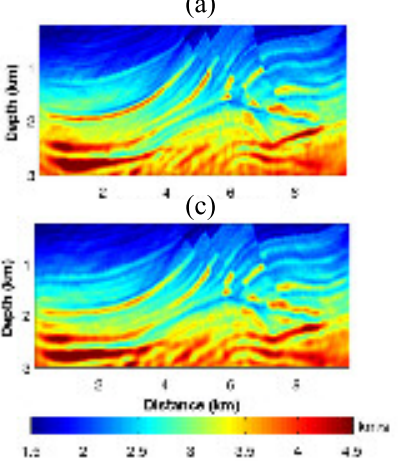

(b)

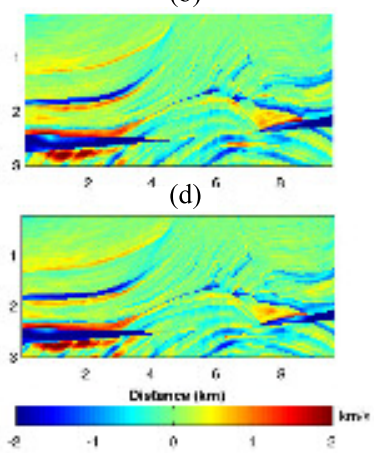

Fig. 12. (a) Hybrid strategy where the inversion is done from 4 to $5 \mathrm{~Hz}$ with the triangular IP and after, this result is used as initial model for the L2-norm OF from $5 \mathrm{~Hz}$. (b) Velocity difference between (a) and the reference true model. (c) and (d) Similar to (a) and (b), but using the normalized signal as an initial model.

the L2 is not the most robust OF [see Fig. 11(k) and (1)]. Its success strongly depends, among other factors, on the accuracy of an initial model to overcome cycle skipping at the lowest inverted frequencies.

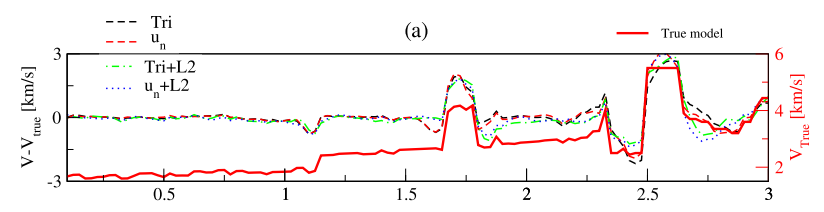

(b)

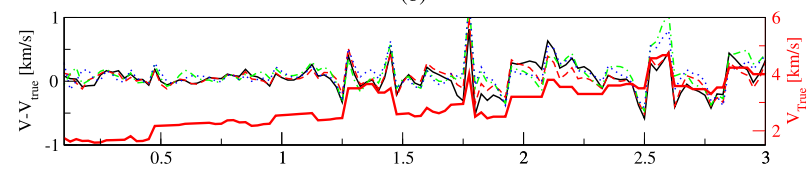

(c)

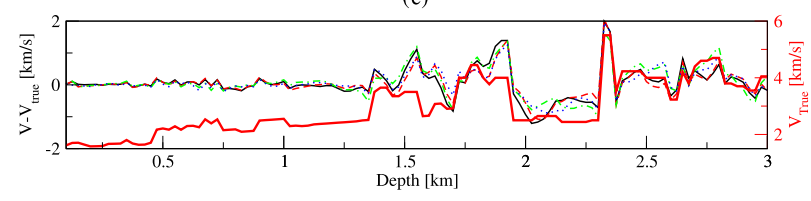

Fig. 13. Depth profiles along the model at $\mathrm{V} 1=2.5, \mathrm{~V} 2=5$, and $\mathrm{V} 3=7.5 \mathrm{~km}$, for the successful inversion strategies shown in Figs. 11 and 12 for the specific OFs: triangular IP (black solid line), normalized signal (red dashed line), "triangular IP + L2" (green dashed dotted line), and "normalized signal + L2" (blue dotted line).

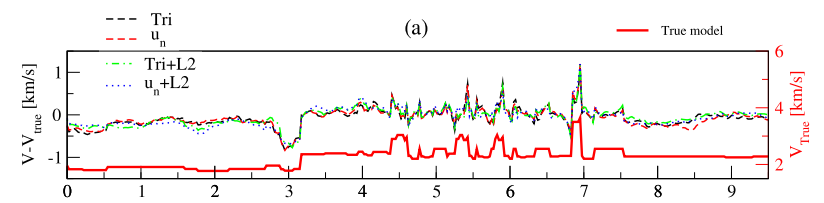

(b)

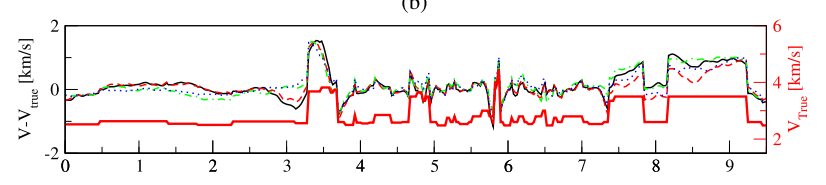

(c)

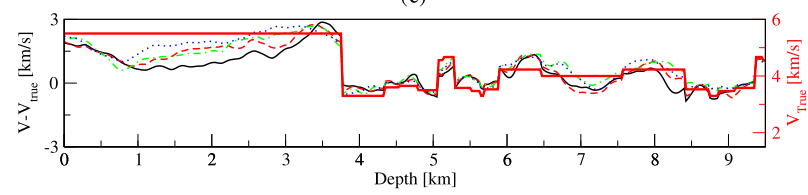

Fig. 14. Horizontal profiles at different depths $\mathrm{H} 1=1, \mathrm{H} 2=1.5$, and $\mathrm{H} 3=2.5 \mathrm{~km}$, for the successful inversion strategies shown in Figs. 11 and 12 for the specific OFs: triangular IP (black solid line), normalized signal (red dashed line), "triangular IP + L2" (green dashed dotted line), and "normalized signal + L2" (blue dotted line).

The alternative measures of the IP functions presented, i.e. the triangular IP and the normalized signal, are robust in the absence of low frequencies in the data. However, as explained in the methodology section, their usage is restricted to monocomponent data, obtained in our work with narrow bandpass filters on the source and the data. In order to obtain a robust strategy with less numerical demands, it is possible to design hybrid strategies using first the IP-based functions for the lower frequencies available in the data. After this initial step, the resulting velocity model may be used as initial model for a L2-norm-based AWI scheme, saving computational resources and time and, more importantly, avoiding numerical errors which might arise when dealing with noisy field data. When using the L2-norm OF, wider band data sets can be applied to source and data, as there is no requirement to work with monocomponent data. In this case, the bandpass also increases $0.5 \mathrm{~Hz}$ at each frequency step but the initial frequency is kept constant. As a summary, the hybrid strategy tested in 
this analysis is:

1) Triangular IP/normalized signal: [4-4.5], [4.5-5] Hz.

2) L2-norm between waveforms: [5-5.5], [5-6] Hz...

Due to the wider band passed data at each frequency step once the L2-norm is applied, the hybrid multiscale strategy is more efficient compared to using the single IP-based OFs, as data are redundant. In Fig. 12, we show the results of the two hybrid strategies in the first column and their corresponding velocity difference in the second column, showing similar results compared with the single $\mathrm{OF}$ strategies. For a better comparison between the single and the hybrid strategies, we plot the depth and horizontal profiles of the velocity difference $\left(V_{\mathrm{AWI}}-V_{\text {True }}\right)$, in Figs. 13 and 14. All the strategies (left $y$-axis) behave in a similar manner; the velocity difference concentrates in the areas where velocity contrasts/gradients are stronger. For a better understanding of this fact, we also plot the $p$-wave velocity of the reference model for the chosen lines in both figures (red solid line), whose correspondent $y$-axis is placed at the right part of the graph. In general, the results worsen with the depth, as shown in the depth profiles in Fig. 13, where the results clearly deteriorate deeper than $1.5 \mathrm{~km}$, for all the chosen lines (V1, V2, and V3) along the horizontal axis. For the same reason, in the horizontal profiles in Fig. 14, the velocity difference in all the strategies for the shallower line $\mathrm{H} 1$ (placed at $1-\mathrm{km}$ depth) are smaller than for H2 (1.5-km depth), and for H3 (2.5-km depth), where all the velocity differences dramatically increase.

\section{CONCLUSION}

We have performed a theoretical and practical analysis on how to measure the IP from a seismic waveform so that it can play an efficient role as OF in the AWI method. The principal value of the IP is not uniquely defined; therefore, we have extended the analysis of the IP in its different domains, wrapped between $(-\pi, \pi)$ and $(-(\pi / 2),(\pi / 2))$ and unwrapped. The waveform can be considered as a sinetype (case A) or as a cosine-type (case B) function. These different criteria give equivalent IP functions but shifted $\pi / 2$ radians with respect to each other. Thus, we explore the results obtained for the different angular domains and for cases A and B. The extraction of the IP from the waveform is done using the Hu-T numerical adaptive data method, which can be easily applied in any type of data whenever it is monocomponent. In our case, the monocomponent character is obtained applying to data and source bandpass filters with $0.5-\mathrm{Hz}$ width.

The wrapped IP functions naturally present phase jumps which need to be treated because they reflect in the inversion results giving noisy images. When smoothing filters are applied to the wrapped IP functions, the numerical errors vanish and good results can be obtained when using data containing low frequencies, just in the cases where the phase discontinuities are time coincident with the extrema of the waveform. This happens for case B when the IP is wrapped between $(-\pi, \pi)$ and for case $\mathrm{A}$ when it is wrapped between $(-(\pi / 2),(\pi / 2))$. In the mentioned works where the wrapped IP is applied in AWI for global earthquake data [3], [9], the IP is calculated in case B with its principal value defined between $(-\pi, \pi)$, coincident as well with our best results. In large scale studies, the presence of low frequencies in the recorded data favors that simulated data are not cycle-skipped, and according to what was shown in our tests, the treatment of the phase jumps' discontinuities helps to obtain good results. But when data lack low frequencies, the waveforms of the initial and reference models are cycle-skipped and this technique does not work properly, as we show in our results. With respect to the unwrapped IP, it produces a linear and a continuous OF; however, it does not work well at minimizing the misfit between the synthetic and the reference data. As it can be observed, the corresponding AS function does not give sufficient details in the description of the waveform.

The main conclusion of our tests is that an appropriate description of the waveform with an IP-based function requires the use of a continuous and a periodic signal, i.e., wrapped, whose extrema are time coincident with the extrema of the original signal. Following this description, it is possible to formulate alternative ways to measure the IP indirectly. Two examples of IP-based OFs that provide robust AWI results even in the absence of low frequencies in the input data are: 1) the triangular IP function, acquired as the original IP wrapped between $(-(\pi / 2)(\pi / 2))$ in case A multiplied by the sign of its quadrature [14] and 2) the normalized signal, firstly proposed in this paper and whose meaning corresponds to the carrier phase function of the waveform. In contrast to the rest of the IP-based OF, the normalized function parameter is uniquely defined between its natural limits and equivalent for the criteria cases A and B. The alternative OFs proposed have the same periodicity as the waveform and do not need any modification to be used directly as OF in AWI, even without low frequencies in the source and the data, and resulted more robust compared with the original wrapped IP parameter. The results between the triangular IP and the normalized signal OFs are similar, as their corresponding ASs share the main features, having the different maxima and minima located at the same time positions.

We have given special emphasis to simplifying the numerical calculation of the AS. We have tested three types of inversions depending on which part of the AS function is solved, $T_{1}$ and $T_{2}$ terms separately and the complete expression $T_{1}+T_{2}$. We have checked that the $T_{2}$ term does not improve the results for the IP OFs working well for data with and without low frequencies, the triangular IP function (case A) and the normalized signal. The success mainly depends on using the $T_{1}$ term in the AS. The $T_{2}$ term for these cases is much smaller compared with the $T_{1}$ and requires a more complex numerical calculation. On the top of that, the calculation of $T_{2}$ is computationally more expensive as might increase the time cost per iteration from $25 \%$ to $60 \%$ compared with using only the $T_{1}$ term. Therefore, we just considered the $T_{1}$ term in the AS function for the selected IP-based OFs.

The method can be improved using the inversion results of the alternative IP-based OFs after one or two inversion steps at the lowest frequencies available in the data as initial models for the traditional L2-norm OF, which is then used for the following inversion steps. Although the use of these hybrid strategies gave similar results as the usage of the single 
IP-alternative OFs, the advantage lies in improving the data redundancy, diminishing computational time, and numerical problems that need to be tackled when extracting the IP from the waveform and calculating its AS function.

\section{ACKNOWLEDGMENT}

The authors would like to thank the anonymous reviewers whose comments/suggestions helped to improve and clarify this paper. They would also like to thank D. Dagnino for fruitful discussions about the adjoint method. This paper is the contribution of the Barcelona Center for Subsurface Imaging.

\section{REFERENCES}

[1] A. E. Barnes, "A tutorial on complex seismic trace analysis," Geophysics, vol. 72, no. 6, pp. W33-W43, 2007.

[2] E. Bedrosian, "A product theorem for Hilbert transforms," Proc. IEEE, vol. 51, no. 5, pp. 868-869, May 1963.

[3] E. Bozdağ, J. Trampert, and J. Tromp, "Misfit functions for full waveform inversion based on instantaneous phase and envelope measurements," Geophys. J. Int., vol. 185, no. 2, pp. 845-870, 2011.

[4] A. J. Brenders and R. G. Pratt, "Full waveform tomography for lithospheric imaging: Results from a blind test in a realistic crustal model," Geophys. J. Int., vol. 168, no. 1, pp. 133-151, 2006.

[5] C. Bunks, F. M. Saleck, S. Zaleski, G. Chavent, "Multiscale seismic waveform inversion," Geophysics, vol. 60, no. 5, pp. 1457-1473, 1995.

[6] B. Chi, L. Dong, and Y. Liu, "Full waveform inversion method using envelope objective function without low frequency data," J. Appl. Geophys., vol. 109, pp. 36-46, Oct. 2014.

[7] D. Dagnino, V. Sallarès, and C. R. Ranero, "Scale- and parameteradaptive model-based gradient pre-conditioner for elastic full-waveform inversion," Geophys. J. Int., vol. 198, no. 2, pp. 1130-1142, 2014.

[8] D. Dagnino, V. Sallarès, B. Biescas, and C. R. Ranero, "Fine-scale thermohaline ocean structure retrieved with 2-D Prestack full-waveform inversion of multichannel seismic data: Application to the Gulf of Cadiz (SW Iberia)," J. Geophys. Res., Oceans, vol. 121, no. 8, pp. 5452-5469, 2016.

[9] A. Fichtner, B. L. N. Kennett, H. Igel, and H.-P. Bunge, "Theoretical background for continental- and global-scale full-waveform inversion in the time-frequency domain," Geophys. J. Int., vol. 175, no. 2, pp. 665-685, 2008.

[10] C. G. Andreu et al., "2D Travel-time tomography of downward continued streamer multichannel seismic data followed by a band-limited full waveform inversion. Application to the Alboran basin (SE Iberia)," Claudia Gras Andreu, Daniel Dagnino, Clara Estela Jiménez Tejero, Adrià Meléndez, Valentí Sallarès and César Ranero, Geophys. Res. Abstracts, Tech. Rep. EGU2017-14250, 2017, vol. 19.

[11] L. Grippo and S. Lucidi, "A globally convergent version of the Polak-Ribière conjugate gradient method," Math. Programm., vol. 78, no. 3, pp. 375-391, 1997.

[12] N. E. Huang, Z. Wu, S. R. Long, K. C. Arnold, X. Chen, and K. Blank, "On instantaneous frequency," Adv. Adapt. Data Anal., vol. 1, no. 2, pp. 177-229, Apr. 2009.

[13] N. E. Huang and Z. Wu, "A review on Hilbert-Huang transform: Method and its applications to geophysical studies," Rev. Geophys., vol. 46, no. 2, p. RG2006, 2008, doi: 10.1029/2007RG000228.

[14] C. E. Jiménez-Tejero, D. Dagnino, V. Sallarès, C. R. Ranero, "Comparative study of objective functions to overcome noise and bandwidth limitations in full waveform inversion," Geophys. J. Int., vol. 203, no. 1, pp. 632-645, 2015, doi: 10.1093/gji/ggv288.

[15] J. Kormann et al., "Acceleration strategies for elastic full waveform inversion workflows in 2D and 3D," Comput. Geosci., vol. 21, no. 1, pp. 31-45, 2017.

[16] P. Lailly, The Seismic Inverse Problem as a Sequence of Before Stack Migrations. Philadelphia, PA, USA: SIAM, 1983, pp. 206-220.

[17] Y. Luo and G. Schuster, "Wave-equation traveltime inversion," Geophysics, vol. 56, no. 5, pp. 645-653, 1991

[18] J. Nocedal and S. J. Wright, Numerical Optimization. New York, NY, USA: Springer-Verlag, 2006, doi: 10.1007/978-0-387-40065-5.

[19] A. H. Nuttall and E. Bedrosian, "On the quadrature approximation to the Hilbert transform of modulated signals," Proc. IEEE, vol. 54, no. 10 , pp. 1458-1459, Oct. 1966

[20] D. Pasalic and R. McGarry, "Convolutional perfectly matched layer for isotropic and anisotropic acoustic wave equations," in Proc. SEG Tech. Program Expanded Abstracts, 2010, pp. 2925-2929.
[21] G. Provenzano, M. E. Vardy, and T. J. Henstock, "Pre-stack full waveform inversion of ultra-high-frequency marine seismic reflection data," Geophys. J. Int., vol. 209, no. 3, pp. 1593-1611, 2017.

[22] D. Subrahmanyam and P. H. Rao, "Seismic attributes-A review," in Proc. Int. Conf. Expo. Petroleum Geophys., Hyderabad, India, 2008, pp. $398-404$.

[23] M. T. Taner and R. E. Sheriff, "Application of amplitude, frequency, and other attributes to stratigraphic and hydrocarbon determination: Application of seismic reflection configuration to stratigraphic interpretation," in M 26: Seismic Stratigraphy_Applications to Hydrocarbon Exploration, vol. 26, C. E. Payton, Ed. Tulsa, OK, USA: American Assotiation of Petroleum Geologist, Jan. 1977, sec. 2, pp. 301-327.

[24] M. T. Taner, F. Koehler, and R. E. Sheriff, "Complex seismic trace analysis," Geophysics, vol. 44, no. 6, pp. 1041-1063, 1979.

[25] A. Tarantola, "Inversion of seismic reflection data in the acoustic approximation," Geophysics, vol. 49, no. 8, pp. 1259-1266, 1984.

[26] J. Virieux, " $P-S V$ wave propagation in heterogeneous media: Velocitystress finite-difference method," Geophysics, vol. 51, no. 4, pp. 889-901, 1986.

[27] J. Virieux and S. Operto, "An overview of full-waveform inversion in exploration geophysics," Geophysics, vol. 74, no. 6, pp. WCC1-WCC26, 2009.

[28] R. E. White, "Properties of instantaneous seismic attributes," Leading Edge, vol. 10, no. 7, pp. 26-32, 1991.

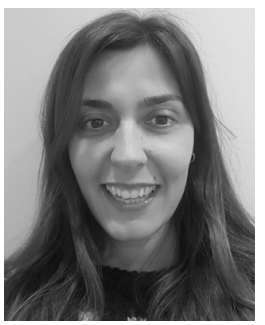

Clara Estela Jiménez Tejero received the bachelor's degree in particle physics from the University of Santiago de Compostela, Santiago, Spain, in 2004, the Diploma degree from the GSI Helmholtz Centre for Heavy Ion Research, Darmstadt, Germany, in 2005, and the M.C. degree in advance physics and the Ph.D. degree in charm hadronic physics from the University of Barcelona, Barcelona, Spain, in 2007 and 2012, respectively.

Since 2013, she has been holding a post-doctora position with the Center for Subsurface Imaging, Marine Sciences Institute, Spanish National Research Council, Barcelona, where she is involved in adjoint waveform inversion problems.

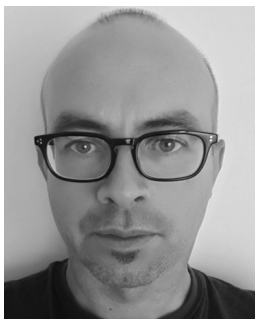

Valenti Sallares received the bachelor's degree in physics, and the M.Sc. and Ph.D. degrees from the University of Barcelona, in 1994, 1996, and 1999, respectively.

He was first a Marie Curie Post-Doctoral Fellow and then a Chargé de Recherche with IRDGéosciences Azur, Villefranche-sur-Mer, France. From 2005 to 2012, he has been a Senior Researcher with the Marine Technology Unit, Spanish CSIC, where he has been with the Institute of Marine Sciences since 2012. He has been principal investigator of 12 competitive research projects funded by regional, national, and European funding agencies and private companies. He has authored or co-authored 57 papers in SCI journals and has over 200 communications in international meetings. His research interests include marine geophysics, especially the methodological development of seismic inversion techniques and applications to study structure and active processes in continental margins.

Dr. Sallarés was a recipient of the García-Siñeriz Award for the Best M.Sc. and the Best Ph.D., in 1997 and 2000, respectively.

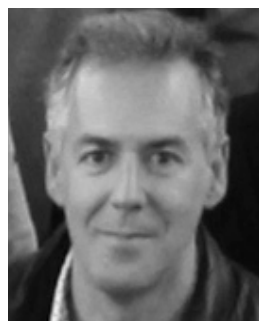

César R. Ranero received the bachelor's degree in geology from the Basque Country University, Leioa, Spain, in 1987, and the Ph.D. degree from the University of Barcelona, Barcelona, Spain, in 1993, from work at the Earth Sciences Institute, Spanish National Research Council (CSIC), Barcelona.

From 1993 to 2005, he was a Researcher with Geomar, Kiel, Germany. He was a Visiting Professor with the French CNRS, Villefranche sur Mer, France, in 2003. From 2005 to 2009, he was the President with the Tectonics and Structural Geology Division, European Geoscience Union, Headquarters in Munich, Germany. Since 2005, he has been an ICREA Research Professor. Since 2007, he has been the Head of the Barcelona Center for Subsurface Imaging, Marine Sciences Institute, CSIC, Barcelona. He has been a (Co-)Chief Scientist of 10 scientific cruises. He was supported by grants from the Spanish Ministry of Industry, the Generalitat de Catalunya, and the European Union. He has authored or co-authored over 100 peer-reviewed papers and book chapters, organized over 20 international meetings, and delivered over 40 invited and keynote talks and seminars in Europe, America, and Japan. 